\title{
Observatorio de Políticas Públicas con enfoque de Derechos Humanos (OPPEDH). Diseño metodológico
}

\section{Observatory of Public Policies with a Human Rights approach (OPPEDH). Methodological design}

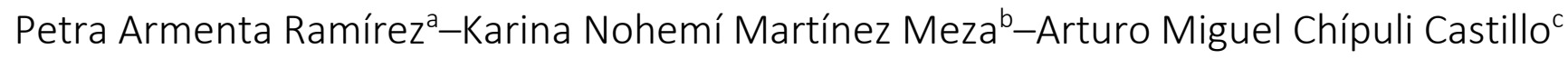 \\ a Responsable del Observatorio de Políticas Públicas con \\ enfoque en Derechos Humanos, Instituto de Investigaciones Jurídicas, \\ Universidad Veracruzana, Xalapa, México. \\ Contacto: parmenta@uv.mx \\ b Observatorio de Políticas Públicas con enfoque en Derechos Humanos, \\ Instituto de Investigaciones Jurídicas, Universidad Veracruzana, \\ Xalapa, México. Contacto: kmartinez@uv.mx \\ c Observatorio de Políticas Públicas con enfoque en Derechos Humanos, \\ Instituto de Investigaciones Jurídicas, Universidad Veracruzana, \\ Xalapa, México. Contacto: achipuli@uv.mx
}

Recibido: 10 de agosto de 2020

Aceptado: 15 de octubre de 2020

RESUMEN: El presente artículo tiene como finalidad mostrar la metodología y los ejes estratégicos que se implementan en el Observatorio de Políticas Públicas con enfoque de Derechos Humanos (OPPEDH) a partir de los enfoques y teorías de la política pública y los derechos humanos. El objetivo general del OPPEDH es crear un espacio para la recolección, generación y sistematización de datos e información sobre políticas públicas con enfoque de derechos humanos en las materias de seguridad pública, desarrollo social, educación y medio ambiente, con un alcance territorial de actuación nacional, estatal y regional que permita disponer a la sociedad de evidencia para estudios cuantitativos y cualitativos.

Palabras clave: Políticas públicas; enfoque de Derechos Humanos; implementación de observatorio. 


\title{
Observatorios - Observatorio de Políticas Públicas con enfoque de Derechos Humanos
}

\begin{abstract}
The purpose of this article is to show the methodology and strategic axes that are implemented in the Observatory of Public Policies with a Human Rights Approach, based on the approaches and theories of public policy and rights humans. The general objective of the OPPEDH is to create a space for the collection, generation and systematization of data and information on public policies with a focus on human rights in matters of public security, social development, education and the environment, with a territorial scope of action national, state and regional that allows the society to have evidence for quantitative and qualitative studies.
\end{abstract}

Keywords: Public Policies; Human Rights Approach; Observatory Implementation.

\section{Introducción}

E sta iniciativa se desarrolla a partir del diagnóstico de pertinencia realizado por algunos investigadores del Instituto de Investigaciones Jurídicas (IIJ) de la Universidad Veracruzana (UV) sobre la implementación de un Observatorio de políticas públicas con el enfoque innovador y necesario de los derechos humanos, mismo que se encuentra vinculado con la misión y objetivos de las líneas de investigación y generación del conocimiento del propio Instituto. El IIJ tiene como misión generar y difundir conocimientos de alto valor social, basado en el uso y la innovación de la ciencia y la tecnología. De igual manera, tiene como objetivos fortalecer el desarrollo de los recursos humanos, la organización institucional, la vinculación con los diferentes sectores de la sociedad, la articulación investigación-docencia, difusión cultural y extensión universitaria, así como instaurar progresivamente nuevos métodos de trabajo, que respondan a la necesidad de convertir a la investigación en la actividad que articule las demás funciones sustantivas de la Universidad Veracruzana.

Para el cumplimiento de estos objetivos, el Instituto contempla entre sus líneas de investigación y generación del conocimiento, la de Derechos Humanos, Gobierno y Políticas Públicas, la cual se enfoca en el estudio de los procesos, actores, marco jurídico, contextos políticos, institucionales, sociales y administrativos de las políticas públicas, desde la óptica de los derechos humanos.

Derivado de lo anterior, y tomando en consideración los resultados de las evaluaciones de políticas públicas en México con enfoque de derechos humanos, nace la iniciativa de crear un observatorio encaminado a generar, recopilar, sistematizar y analizar los datos e información relativas a las temáticas de seguridad pública, educación, desarrollo social y medio ambiente, con un período de implementación de 3 años cada uno. 
El presente artículo tiene como finalidad realizar una breve descripción de la metodología y ejes estratégicos que orientan el desarrollo de las funciones que se llevan a cabo en el mismo. Motivo por el cual, el documento se divide en cinco apartados: 1. Objetivo, misión y visión del observatorio; 2. Justificación; 3. Enfoques teóricos; 4. Metodología; y 5. Ejes estratégicos.

\section{Objetivo, misión y visión}

El observatorio tiene como objetivo general construir un espacio para la recolección, generación y sistematización de datos e información sobre las políticas públicas con enfoque de derechos humanos en México a nivel nacional, estatal y regional en materia de seguridad pública, desarrollo social, educación y medio ambiente, que permita disponer de evidencia para estudios cuantitativos y cualitativos, y proponer modelos de evaluación de las políticas públicas en sus diferentes etapas. En este sentido, y a fin de lograr lo antes planteado, el Observatorio tendrá cuatro objetivos específicos, mismos que se refieren a continuación:

I) Recabar, generar y sistematizar la información del objeto de estudio para crear una base de datos que será pública y de acceso abierto.

II) Analizar e interpretar los resultados en la recolección de datos como evidencia teórica y empírica para el diagnóstico.

III) Difundir, mediante la participación en foros, talleres conferencias de prensa, así como la publicación de libros, artículos, material informativo en medios impresos, electrónicos y sitio web del observatorio.

IV) Gestionar instrumentos y/o mecanismos de colaboración con instancias de gobierno y con la sociedad civil que permitan canalizar los resultados obtenidos por el observatorio, así como para obtención y generación de información.

En este mismo sentido, el Observatorio de Políticas Públicas con Enfoque de Derechos Humanos tiene como misión constituir un espacio académico, multidisciplinario, de integración interinstitucional e intersectorial para la obtención, análisis, procesamiento, monitoreo y divulgación de la información sobre el enfoque de derechos humanos en las políticas públicas relativas a seguridad pública, desarrollo social, educación y medio ambiente. Cuestión que va de la mano con una visión proyectada hacia el año 2025, donde se 
pretende que el Observatorio de Políticas Públicas con Enfoque de Derechos Humanos implemente con éxito la primera temática (seguridad pública), misma que será un referente confiable, viable y verificable, que orientará, por un lado, a los gobiernos en el diseño, formulación, implementación, y evaluación de políticas públicas en términos de gobernabilidad y gestión pública, y por el otro, contribuirá a la gobernanza democrática y a los objetivos de desarrollo sostenible derivados de la Agenda 2030, en la observancia de las obligaciones en materia de Derechos Humanos. Del mismo modo, se estará actualizando y complementando su base de datos con nuevas investigaciones, aportaciones e información a fin de generar una sinergia multidisciplinaria.

\section{Justificación}

El Observatorio de Políticas Públicas con Enfoque de Derechos Humanos, como parte de la Coordinación Universitaria de Observatorios (CUO), nace de la preocupación fundamental por advertir, con base en datos y evidencia científica, la incidencia de los derechos humanos en las políticas públicas nacionales, estatales y regionales. A partir de la reforma de 2011 en materia de derechos humanos, las autoridades de todos los niveles y órdenes de gobierno, así como de los tres poderes, se encuentran obligadas por mandato constitucional a respetar, proteger, garantizar y promover los DDHH en el marco de sus competencias. Lógicamente, este mandato conlleva la inclusión en las políticas públicas, lo cual motivó un diagnostico situacional general con base en los datos que se presentan a continuación:

1) Que a nivel nacional, hasta octubre de 2018 , se cuenta con 20 diagnósticos y 19 programas estatales de derechos humanos publicados (www.gob.mx) (ver figura 1). Ambos documentos se consideran instrumentos de política pública que definen actividades y metas articuladas para orientar la acción gubernamental en el cumplimiento de las obligaciones de promoción, protección, respeto y garantía de los derechos humanos. No obstante, son pocos los estudios, diagnósticos y evaluaciones que analicen las políticas públicas con enfoque de derechos humanos, aún menos lo son aquellos que analicen la eficacia y eficiencia de dichos programas. 


\section{Programas Estatales de Derechos Humanos}

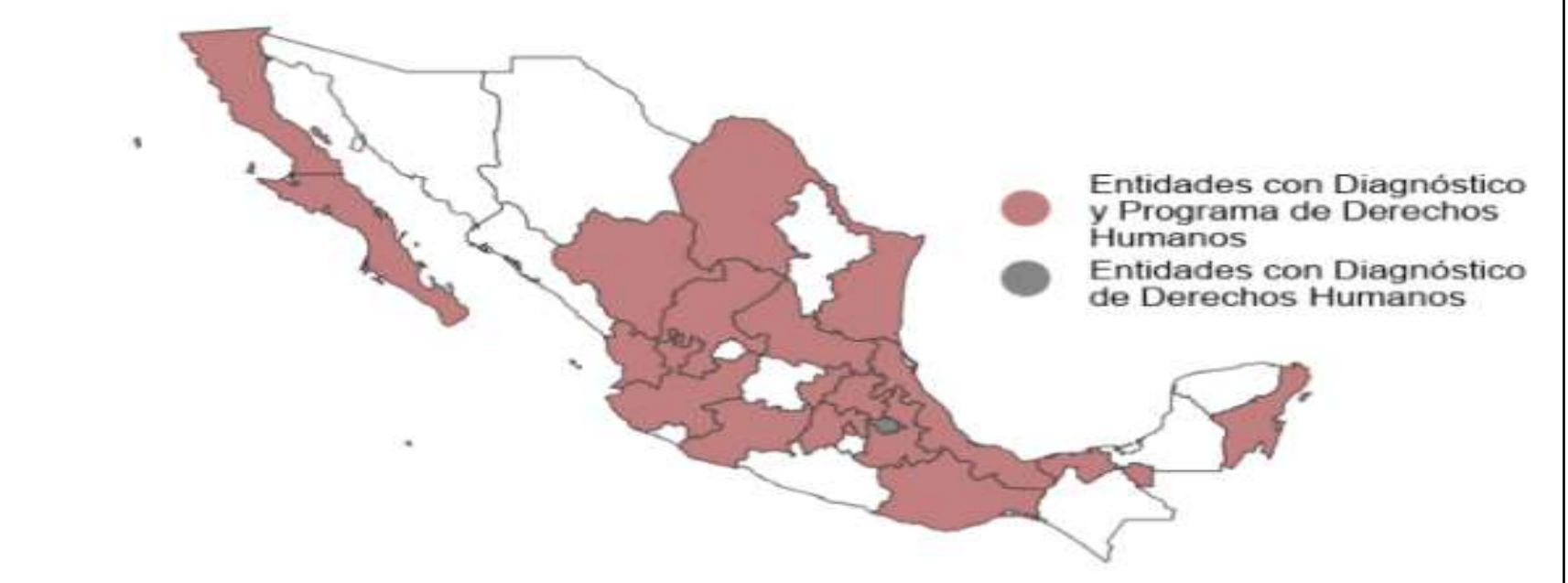

Figura 1. Programas Estatales de Derechos Humanos. Fuente: www.gob.mx/segob/acciones-yprogramas/diagnosticos-y-programas-estatales-de-derechos-humanos

Con base en lo anterior, se observa que a pesar de que existen en las entidades federativas programas y diagnósticos de y en políticas de derechos humanos, no son claros ni definitivos en cuanto a la incidencia de los derechos humanos en cada una de las etapas del ciclo de las políticas públicas (diagnostico, diseño, formulación, implementación, evaluación).

2) Que existe la necesidad de recolectar y sistematizar la información sobre el enfoque de derechos humanos en las políticas públicas relativas a las temáticas de seguridad pública, desarrollo social, educación y medio ambiente.

3) Que existe la necesidad de crear un diagnostico situacional sobre las políticas públicas con enfoque de derechos humanos en México.

4) Se resalta la creación de un modelo metodológico que permita la obtención, procesamiento y presentación de datos sobre las políticas públicas con enfoque de derechos humanos en México.

5) Que es necesaria la implementación de un Observatorio de políticas públicas con enfoque de derechos humanos que traiga consigo el fortalecimiento de la línea de generación y aplicación al conocimiento del Instituto de Investigaciones Jurídicas de la Universidad 


\section{Observatorios - Observatorio de Políticas Públicas con enfoque de Derechos Humanos}

Veracruzana denominada "Derechos humanos, gobierno y políticas públicas" y del programa de Doctorado en Derecho en los rubros de publicación y proyección de tesis, además de contribuir en la actualización de toda persona interesada en temas de materia jurídica y en la formación de los estudiantes de Derecho de nuestra casa de estudios, de cualquier otra institución pública y privada.

\section{Enfoques teóricos}

Los enfoques teóricos que fundamentan el diseño e implementación del Observatorio son el de política pública y el de derechos humanos, el primero está vinculado a las transformaciones de la esfera pública, a la intervención del Estado, a la relación de éste último y el mercado y al modo en que se ha configurado la acción gubernamental. Se relaciona con la capacidad que tiene el gobierno para intervenir racionalmente en la solución de los problemas públicos (Laswell, 1951). Se entiende que la sociedad se torna más compleja y que el gobierno tiene límites económicos, institucionales y normativos, con escenarios políticos plurales y competitivos, por lo que "la tarea de gobernar se orienta a un nuevo marco de innovación gubernamental y de desarrollo de capacidades adecuadas de los agentes tomadores de decisiones" (Parsons, 2007:23).

La política pública se enfoca en el manejo de la información para la generación de evidencia que dé sustento de la articulación del conocimiento y práctica, de la realidad, el diseño e implementación de políticas. Busca explicar "el cómo, el por qué y para qué los gobiernos adoptan determinadas medidas y actúan o no actúan" (Heidenheimer et al, 1990; Parsons, 2007:31). Para ello utiliza un enfoque interdisciplinario para contribuir a la democratización de la sociedad.

Una política pública designa "la existencia de un conjunto conformado por uno o varios objetivos colectivos considerados necesarios o deseables y por medios y acciones que son tratados, por lo menos parcialmente, por una institución u organización gubernamental con la finalidad de orientar el comportamiento de actores individuales o colectivos para modificar una situación percibida como insatisfactoria o problemática" (Roth, 2002:27).

Por su parte, el Enfoque de Derechos Humanos representa un aporte de carácter sustantivo para los contenidos de las políticas públicas que pone énfasis en las obligaciones estatales, consagrándose estas como directrices de contenido, de tal forma que las políticas públicas se vislumbran como medios de satisfacción de derechos, y no solo como mecanismos para la solución de problemas y demandas insatisfechas (Salazar, 2014). 


\section{Metodología}

Por cuanto hace al alcance territorial de actuación del Observatorio, este se circunscribe a las políticas públicas nacionales, estatales y municipales, en las temáticas de seguridad pública, educación, desarrollo social y medio ambiente, que se desarrollan durante las etapas metodológicas descritas a continuación:

Primera etapa: se recolectará, de manera sistemática, los datos cualitativos y cuantitativos sobre los principios, obligaciones y elementos transversales del enfoque de derechos humanos en las políticas públicas, posteriormente se publicarán los resultados que serán de acceso gratuito y abierto, utilizando la herramienta atlas ti.

Segunda etapa: se analizarán e interpretarán los resultados derivados de la recolección de datos para crear un diagnóstico situacional, así como artículos científicos los cuales se divulgarán y comunicarán en medios electrónicos y escritos de modo gratuito y abierto, además se realizarán informes y se llevarán a cabo cursos, talleres y coloquios.

Para incorporar la perspectiva de Derechos Humanos (DH) al análisis de las políticas públicas es necesario tener presentes diversos elementos: a) los Estándares Internacionales de Derechos Humanos y b) los Elementos Transversales. Los Estándares Internacionales parten de la base de que el Derecho Internacional de los Derechos Humanos (DIDH) representa un marco conceptual que es aceptado por la comunidad internacional y que puede ofrecer un sistema coherente de principios y reglas que contribuye a definir con mayor precisión las obligaciones de los Estados frente a los DH (Abramovich, 2006:36).

Este primer elemento, proviene de la adecuada identificación de las obligaciones establecidas en las distintas fuentes del DIDH. Una vez identificados los diversos componentes del derecho, es menester advertir cuáles son las obligaciones específicas insertas en cada uno de ellos: respetar, proteger, garantizar y promover. A partir de estas obligaciones se realiza un desglose de cada uno de estos componentes (ver tabla 1, p. 21) que el DIDH ha señalado como parte del derecho elegido, midiendo el nivel de cumplimiento o incumplimiento del contenido del programa de política pública con los estándares ya señalados. 
Tabla 1.

Obligaciones de los Derechos Humanos

\begin{tabular}{|c|c|c|c|c|}
\hline Obligación & $\begin{array}{c}\text { Objetivo respecto del } \\
\text { DH }\end{array}$ & $\begin{array}{c}\text { Conducta } \\
\text { requerida }\end{array}$ & $\begin{array}{c}\text { Violación } \\
\text { de DH }\end{array}$ & Cumplimiento \\
\hline Respetar & Mantener & Negativa & Positiva & Inmediato \\
\hline Proteger & Mantener & Positiva & Negativa & Inmediato>progresivo \\
\hline Garantizar & Realizar y Mejorar & Positiva & Negativa & Inmediato-Progresivo \\
\hline Promover & Mejorar & Positiva & Negativa & Progresivo \\
\hline
\end{tabular}

Fuente: Elaboración propia a partir de la literatura del Derecho Internacional de Derechos Humanos.

Por su parte, los elementos transversales del enfoque de DH representan principios medulares reconocidos por los tratados internacionales, las observaciones generales de los Comités de Naciones Unidas, así como los informes y la jurisprudencia de los órganos de protección internacional de los DH, y que deben permear en el diseño e implementación de las políticas públicas (Vázquez y Delaplace, 2011:44). Entre estos elementos podemos encontrar al principio de igualdad, la participación, los mecanismos de exigibilidad, la coordinación institucional, la perspectiva de género y la capacitación en DH. Cada uno de los cuales repercutirá en la forma de intervención. (ver tabla 2, p.22). 
Observatorios - Observatorio de Políticas Públicas con enfoque de Derechos Humanos

Tabla 2

Elementos transversales de los Derechos Humanos

\begin{tabular}{|c|c|}
\hline Elemento Transversal & Descripción \\
\hline Participación & $\begin{array}{l}\text { Se trata de mecanismos de participación que generen la posibilidad de que } \\
\text { los ciudadanos y las organizaciones de la sociedad civil puedan incidir } \\
\text { sobre procesos decisorios. }\end{array}$ \\
\hline Principio de Igualdad & $\begin{array}{l}\text { Impacto de la intervención en contextos particulares que, tomando en } \\
\text { cuenta las diferencias y características del grupo o sector, requieren de } \\
\text { determinadas acciones positivas o medidas transformadoras, que permitan } \\
\text { el igual acceso y disfrute de los derechos. }\end{array}$ \\
\hline Coordinación Interinstitucional & $\begin{array}{l}\text { Se trata de la adopción de un enfoque holístico donde las diversas } \\
\text { instancias gubernamentales deben actuar de manera coordinada y } \\
\text { articulada, con el fin de romper con la intersectorialidad y lograr la } \\
\text { integralidad de los diseños y los abordajes para la acción (a diferencia de la } \\
\text { política pública tradicional) de problemas públicos entendidos como } \\
\text { ejercicio de derechos (Salazar, 2014:156 y 157; Vázquez y Delaplace, 2011:47, } \\
\text { Rossi y Moro, 2014:153). }\end{array}$ \\
\hline Mecanismos de Exigibilidad & $\begin{array}{l}\text { Mecanismos o dispositivos institucionales establecidos con la finalidad de } \\
\text { garantizar los derechos humanos dentro del marco de la política pública. } \\
\text { Esta garantía conlleva el establecimiento de instrumentos de naturaleza } \\
\text { política, jurisdiccional, administrativa o a través de órganos autónomos. }\end{array}$ \\
\hline $\begin{array}{c}\text { Educación en Derechos } \\
\text { Humanos }\end{array}$ & $\begin{array}{l}\text { Ésta se concibe, no sólo como la manera de hacer llegar cierta información } \\
\text { (contenidos o datos) a quienes la desconocen, sino que implica un trabajo } \\
\text { ético, crítico y político, centrado en contextos reales y concretos, que } \\
\text { permite la construcción progresiva de una visión sobre la persona y las } \\
\text { relaciones entre estas, basadas en la dignidad y pautas de convivencia } \\
\text { social inclusiva, justa, pacífica y solidaria (Rondino, 1999:7). }\end{array}$ \\
\hline
\end{tabular}

Fuente: Elaboración propia a partir de la literatura del enfoque de derechos humanos.

Llegado a este punto, es importante señalar que el enfoque de derechos humanos en las políticas públicas es un elemento que puede fortalecer e incidir en la evaluación del cumplimiento de los objetivos mundiales de la Agenda 2030 con el modelo de desarrollo sostenible, el cual surge del interés, preocupación y discusión global por preservar la diversidad biológica, disminuir la disparidad de los recursos entre quienes viven actualmente, mejorar la calidad de vida del ser humano y compensar a las generaciones futuras por el menoscabo de la dotación de recursos provocada por la acción y la toma de decisión de las generaciones actuales. Este modelo de desarrollo promueve la prosperidad y las oportunidades económicas, una educación de calidad, un mayor bienestar social, diversas 
formas de asegurar la equidad y dignidad, así como una sociedad pacífica, justa, inclusiva, participativa, comprometida e innovadora.

Así, la implementación del Observatorio iniciará con el tema de seguridad pública a partir del desarrollo de investigaciones cualitativas y cuantitativas que aplicarán los métodos comparativos y estudio de caso. De igual modo, para lograr los objetivos de la investigación y del observatorio, se utilizarán herramientas cualitativas de recolección de información, entre las que destacan entrevistas a profundidad, entrevistas semi-estructuradas, la técnica documental (por medio de informes de gobierno estatal y municipal, documentos oficiales internos, planes de desarrollo estatal y municipal, material de prensa, información solicitada por la Plataforma Nacional Transparencia y notas periodísticas).

Para el análisis y la interpretación de la información se utilizará el software de Atlas ti con la finalidad de sistematizar, contrastar las proposiciones teóricas del estudio con las evidencias obtenidas; e identificar los elementos centrales y patrones. La batería de indicadores a monitorear se definirá en función de la base de datos generada, así como su periodicidad y difusión.

\section{Ejes estratégicos}

Para alcanzar la misión y la visión planteadas, el Observatorio de Políticas Públicas con Enfoque de Derechos Humanos ha definido cuatro ejes estratégicos, mismos que habrán de ejecutarse desde el inicio de sus operaciones y que son:

a) Recopilación, generación e Integración de datos e información para la conformación de una plataforma de datos actualizada y sistematizada de fácil manejo.

b) Análisis y presentación de los datos e información.

c) Intercambio y difusión de la información obtenida de la materia de estudio.

d) Gestión operativa del observatorio.

\section{Conclusiones}

El Observatorio de Políticas Públicas con Enfoque de Derechos Humanos parte de la necesidad imperante de contar con bases de datos solidas respecto de la incidencia del 
enfoque de derechos humanos en las políticas públicas nacionales, regionales y estatales, derivado de lo cual puede ser posible advertir el grado de cumplimiento/incumplimiento de las obligaciones generales señaladas por el corpus iuris internacional de los derechos humanos, y marcar una ruta de reformulación de la acción gubernamental con la finalidad de hacer patente el cumplimiento de los derechos humanos en todos los ámbitos de la acción pública. En este tenor, el OPPEDH materializa el cumplimiento social e institucional del Instituto de Investigaciones Jurídicas de la Universidad Veracruzana con el Estado de Derecho y la Democracia.

\section{Referencias}

Abramovich, V. (2006). Una aproximación al enfoque de derechos en las estrategias y política de desarrollo, Revista de la CEPAL, (88), 35-50.

Lasswell, H. (1951/1992). La orientación hacia las políticas. En Aguilar V. L., (Ed.). El estudio de las políticas públicas (pp. 79-103) México: Porrúa.

Parsons, W. (2007). Políticas Públicas. Una introducción a la teoría y la práctica del análisis de políticas públicas. En Miño y Dávila (Ed), Facultad Latinoamericana de Ciencias Sociales. Madrid: FLACSO

Rodino, A.M. (1999). La educación en valores entendida como educación en derechos humanos. Sus desafíos contemporáneos en América Latina, Recuperado de: http://www.juridicas.unam.mx/publica/librev/rev/iidh/cont/29/pr/pr7.pdf.

Rossi, J. y Moro, J. (2014). Ganar Derechos. Lineamientos para la formulación de políticas públicas basadas en derechos humanos, Argentina, Instituto de Políticas Públicas en Derechos Humanos del MERCOSUR, Serie Documentos de Trabajo 2.

Roth, D. (2002). Políticas públicas. Formulación, implementación y evaluación. Bogotá: Ediciones Aurora.

Salazar, P. (coord.) (2014). "Chapter 7. Reform and Public Policies" in the Constitutional Reform on Human Rights: A Conceptual Guide, Mexico, Instituto Belisario Domínguez: 142-175.

Vázquez, D., y Delaplace, D. (2011). Políticas públicas con perspectiva de derechos humanos. Un campo en construcción, Sur. Revista Internacional de Derechos Humanos, 8(14), 3564. 